\title{
A study of abnormal muscular patterns in diverticular disease of the colon using the polysiloxane foam enema
}

\author{
L. E. HUGHES \\ From the Department of Surgery, University of Queensland, and the Royal Brisbane Hospital, \\ Brisbane, Australia
}

SUMMARY Three-dimensional, functional studies of the left colon using a polysiloxane foam enema have shown that three types of impressions due to muscular contractions are commonly found in the region of the rectum and sigmoid, and that at least two of these are closely associated with the presence of diverticular disease.

Each type of abnormality has been defined and its extent and frequency assessed. A tentative mechanism for the development of the main muscular abnormality through muscle thickening and simple sigmoid curves is put forward, and it is suggested that the third type of abnormality may be associated with sphincteric action at the recto-sigmoid junction.

The reproducibility of findings on repeated examinations must mean that there is a local anatomical basis for the muscular impressions seen and that such contractions do not represent evanescent peristaltic type activity.

Sigmoid resection and myotomy as practised for diverticular disease should eliminate the first type of abnormality in the majority of cases, 'but', it is necessary to carry the operation well distally to ensure this. Neither operation is likely to correct the type III abnormality, and further work is necessary to determine whether this is responsible for some of the symptoms persisting after surgery.

Diverticulosis of the colon as a cause of inflammatory disease and a mimic of carcinoma has been well recognized throughout this century. As management of gross diverticulitis by resection has become standardized, there has been a shift of interest toward a group of syndromes which appear to be due to functional disorders of the colon. The finding that in many cases of sigmoid resection for 'diverticulitis' inflammatory change has been minimal or absent while thickening of the muscular coat has been conspicuous (Morson, 1963) has led Reilly to treat diverticulitis by a long myotomy of the sigmoid colon rather than resection (Reilly, 1966). In many ways this substitution of myotomy for resection seems to be a logical and desirable development, 
but at present a sound understanding of the clinical and pathological principles inherent in this operation is lacking.

Two particular deficiencies are obvious. Little is known of the natural history of 'functional' symptoms of the colon and very little of the nature of the muscle abnormality itself. Although clearly recognized in its advanced state, the abnormality has not been defined, nor have its incidence, extent, and relation to diverticula or to clinical symptoms been described. Some of these latter factors have been examined in vitro by a histological examination of necropsy colons (Hughes, 1968).

The purpose of the work reported here has been to carry out a functional study of the muscle of the left colon, using the polysiloxane foam enema ${ }^{1}$ (Cook and Margulis, 1961). This material, when introduced into the bowel, forms a plastic cast which outlines the colon as far as the splenic flexure, so that the main area involved in diverticular disease is well shown.

Muscular changes demonstrated by this method have been correlated with the presence of diverticular disease, but in the present study no attempt has been made to relate these changes to specific symptom complexes.

\section{Materials and Methods}

PATIENTS

Fifty colonic casts from 46 patients were studied in this project. The 46 patients comprised a consecutive series of successful foam enema examinations, and the indications for the examinations were as follows. Forty-three patients (four of whom had two examinations) were undergoing clinical investigation for symptoms suggestive of a colonic neoplasm. All had previously undergone at least one barium enema examination, with a negative or equivocal report with reference to malignancy. Three patients who had undergone sigmoid colectomy two years earlier for vesico-colic fistula (two cases) and perforated diverticulitis (one case) were examined to determine the extent of routine sigmoid colectomy in relation to the muscular abnormality reported in this paper. Evaluation of the primary muscle abnormality could not be carried out in these three post-resection patients.

\section{EXAMINATION TECHNIQUE}

Two hours after preparation with a tap water bowel washout, a Sigmoidform enema examination was carried out with the patient in the erect position. One hundred and twenty-five $\mathrm{g}$ of catalysed liquid polymer was inserted through a large rectal balloon catheter. After a period of four minutes, to allow the polymer to set to a soft plastic consistency, the patient was allowed to evacuate the enema.

\section{ASSESSMENT OF CASTS}

All casts were analysed for specific types of muscular impression. It was found that three types of muscular contraction could be clearly recognized, and these were designated types $I$, II, and III. The presence and extent of these muscle changes were recorded, measurements being taken from the tip of the catheter, which leaves a clear impression on the cast. (The cast does not extend to the anal verge, as the ampulla of the rectum is occupied by the balloon catheter. The tip of the catheter lies at a point approximately $15 \mathrm{~cm}$ from the anal verge.)

The barium enema films from the same patients were then examined independently for the presence of diverticula and muscle abnormalities. The presence or absence of diverticula was determined from combined evaluation of the polysiloxane and barium enemas. This allowed the determination of the relationship of the individual types of muscle change to each other and to the presence of diverticula.

\section{Results}

TYPES OF MUSCULAR IMPRESSIONS SEEN

\section{Type I}

The 'typical' muscle abnormality of diverticular disease (Fig. 1) consisted of deep impressions due to muscular bands alternating from side to side. Diverticula were frequently seen arising from the apex of the ridges between the bands. This finding corresponds to the pathological picture shown in Figure 2. This abnormality was present in 23 of the 43 patients available for assessment. It began at a mean distance of $18 \mathrm{~cm}$ above the tip of the catheter, ie, approximately $33 \mathrm{~cm}$ from the anal verge, with a range of 7.5 to $30 \mathrm{~cm}$. The mean length of the abnormal area itself was $12 \mathrm{~cm}$, with a range of $6 \mathrm{~cm}$ to $20 \mathrm{~cm}$.

\section{Type II}

A second type of muscle abnormality seen was that of a series of 'sigmoid curves', usually four or five alternating convexities and concavities (Fig. 3). This change was sometimes present as an isolated finding, but it also occurred below an area of type I change, as seen in Figure 3. In this case the two were usually in direct conti- 

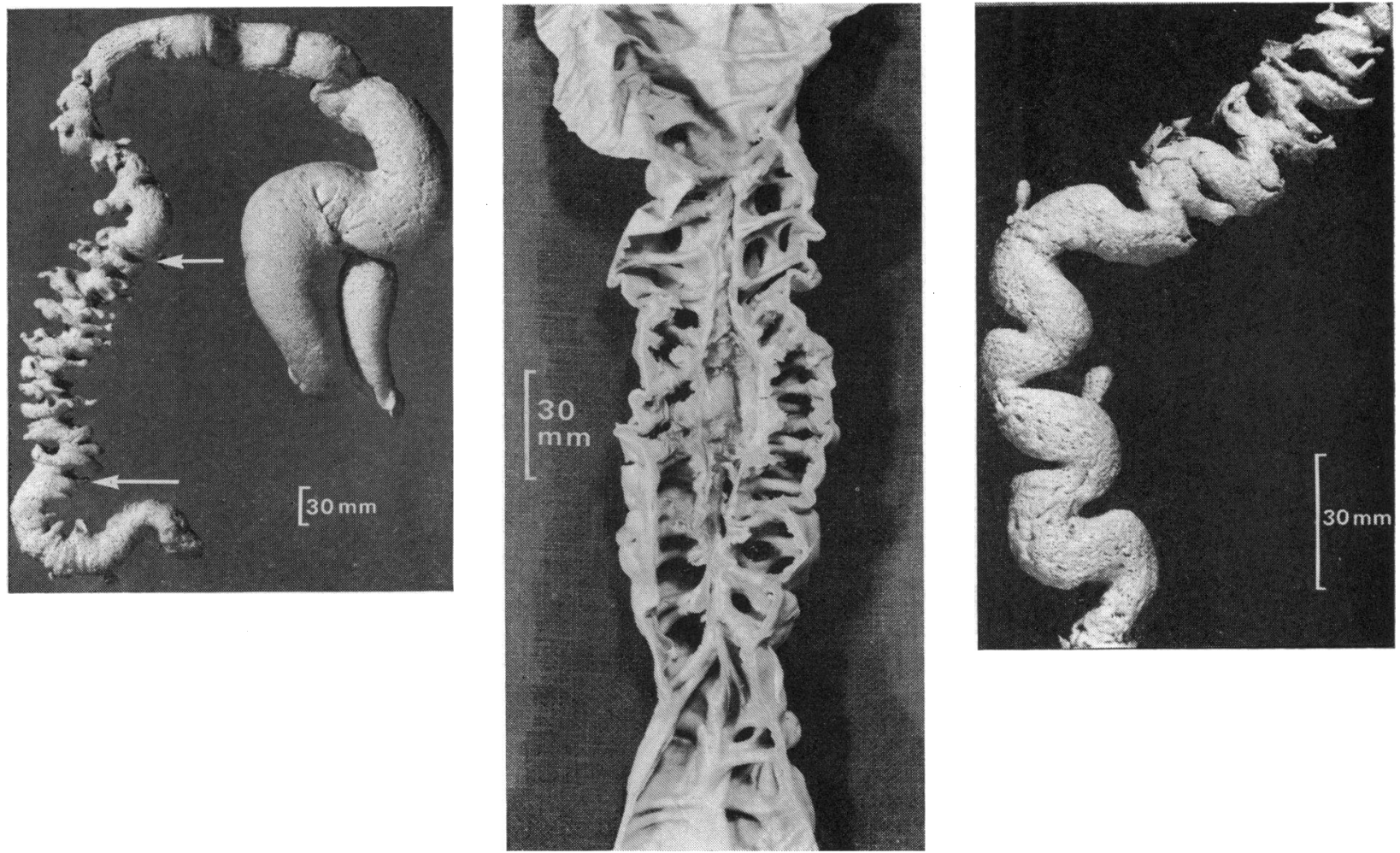

Fig. 1 Foam enema cast of rectum and sigmoid showing typical appearance of type I muscle abnormality (left). Area involved shown between arrows.

Fig. 2 Sigmoid colon involved with diverticular disease opened to show type I muscle abnormality and diverticula arising between the muscle bands (centre).

Fig. 3 Foam enema cast showing the sigmoid curves of type II muscle abnormality in the lower half, merging into type I abnormality in the upper half (right).

nuity, but sometimes a length of normal bowel was interposed between them. This type of muscle change was seen in 14 of 43 patients. On two occasions two separate areas of type II change were seen in the recto-sigmoid colon, separated by a length of normal bowel. In some cases this abnormality appeared to be shortened by longitudinal contraction, giving rise to an asymmetry which gradually merged into a picture closely resembling type I change (Fig. 4).

\section{Type III}

The third change noted was one where two or three deep muscular contractions occurred close together, but differed from type II muscle change in that the bands were on the same side of the bowel, instead of alternating from side to side. In addition, the change was much more localized than was seen with type II. The bands were usually in the concavity of the recto-sigmoid bend, but were rarely seen on the convexity. Not infrequently large diverticula arose from the ridges between them (Fig. 5), suggesting that high pressures were developed in this region. Type III change occurred much lower than types I and II and was usually seen as a localized entity at the recto-sigmoid junction, but occasionally it was present at two sites in this region, separated by a short length of normal bowel. It was present in 12 colons in this series.

COMPARISON OF FINDINGS IN SIGMOIDFORM AND BARIUM ENEMA EXAMINATIONS The findings on Sigmoidform and barium enemas are summarized in Figure 6. Diverticula could be seen in the barium enema in 26 of the 29 cases in which they were shown to be present, 


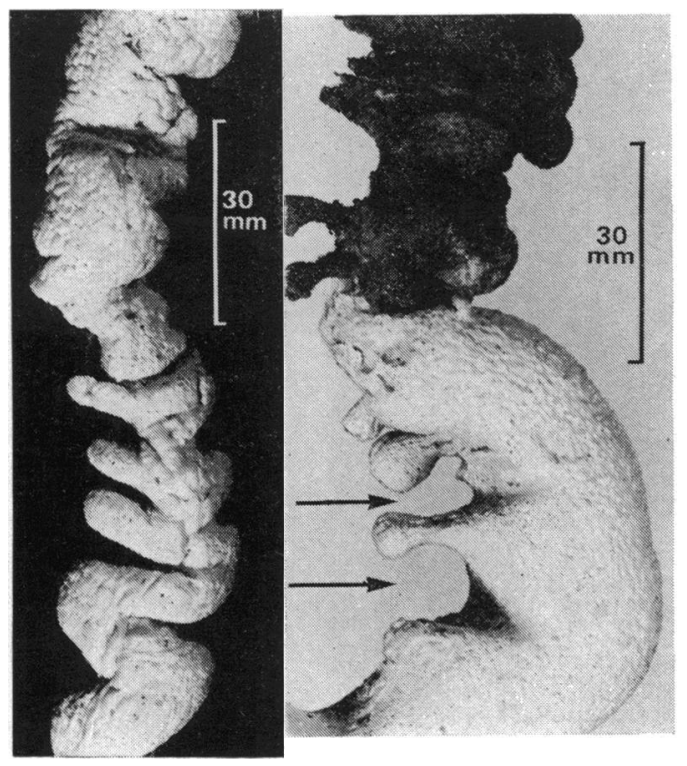

Fig. 4 Foam enema cast (left) showing type II muscle abnormality below a segment with an appearance which suggests an intermediate form between types II and I abnormality. The form of this segment suggests that type II abnormality may have been 'concertinad' by longitudinal spasm.

Fig. 5 Foam enema cast (right) showing type III muscle abnormality. Large diverticula can be seen arising adjacent to the deep muscular impressions (arrows).

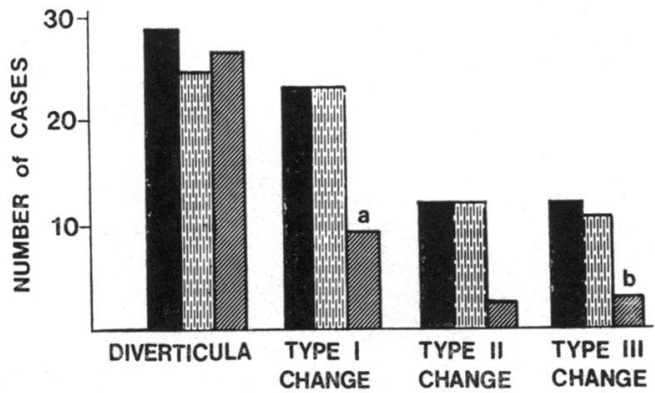

and in the Sigmoidform enema in 25 of the 29 cases. In assessing muscular change, the Sigmoidform enema was more accurate than the barium enema, as frequently muscular change could be clearly seen in the silicone foam enema, while no such change could be seen in the corresponding barium enema. In some such cases, the barium enema films suggested the presence of muscular change, but overlapping loops of bowel and other technical factors precluded a confident diagnosis.

ASSOCIATIONS BETWEEN DIVERTICULOSIS AND THE VARIOUS TYPES OF MUSCLE CHANGE

The association between the presence of diverticulosis (as determined from combined assessment of the silicone foam and barium enemas) and the three types of muscle change is set out in Table I. It is shown that there is a significant association between diverticulosis and change of types I and II change but not between diverticulosis and type III change.

The association of type I change with that of types II and III is set out in Table II. Types I and II show an association at a significant level, but the presence of types I and III does not appear to be associated. 


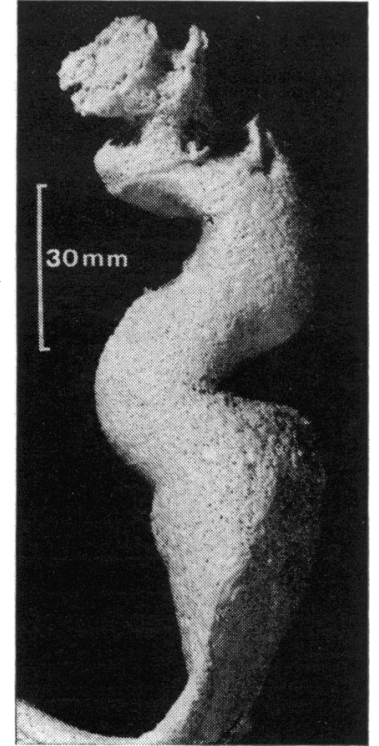

Fig. 7 Cast of upper rectum and lower sigmoid from a patient who underwent sigmoid colectomy two years previously for diverticulitis. Three persistent type I muscle bands are seen at the upper end of the cast, which has been interrupted at the level of the anastomosis.

\begin{tabular}{lrlll}
\hline Charge & $\begin{array}{l}\text { Incidence of } \\
\text { Diverticulosis }\end{array}$ & $\chi(I)^{2}$ & P \\
\hline Type I present & $22 / 23$ & $(96 \%)$ & & \\
Type I absent & $7 / 20$ & $(35 \%)$ & 15.30 & $<0.001$ \\
Type II present & $13 / 14$ & $(93 \%)$ & & \\
Type II absent & $16 / 29$ & $(55 \%)$ & 7.54 & $<0.01$ \\
Type III present & $9 / 12$ & $(75 \%)$ & & \\
Type III absent & $20 / 31$ & $(64 \%)$ & 0.52 & $>0.1$ \\
All cases & $29 / 43$ & $(67 \%)$ & & \\
\hline
\end{tabular}

Table I Association between diverticulosis and the three types of muscle change ${ }^{1}$

${ }^{1}$ Fourteen of the 43 patients studied were considered by the polysiloxane foam enema to have no muscular abnormality. Polysiloxane enema casts from normal colons most commonly show a smooth contour without indentations of any kind. Occasionally a few regular indentations occur along one side of the bowel; such indentations are not constant from one examination to another.

\begin{tabular}{lrrrr}
\hline Change & $\begin{array}{l}\text { Incidence of } \\
\text { Type I Change }\end{array}$ & $\chi(I)^{2}$ & P \\
\hline $\begin{array}{l}\text { Type II present } \\
\text { Type II absent }\end{array}$ & $13 / 14$ & $(93 \%)$ & & \\
Type III present & $10 / 29$ & $(34 \%)$ & 10.75 & $<0.01$ \\
Type III absent & $8 / 12$ & $(66 \%)$ & & \\
& $15 / 31$ & $(48 \%)$ & 0.47 & $>0.1$ \\
All cases & $23 / 43$ & $(53 \%)$ & & \\
\hline
\end{tabular}

Table II Association between types II and III muscle change and type I muscle change

\section{REPRODUCIBILITY OF FINDINGS}

Repeat examinations have been carried out in a number of patients including four of those in this series. A remarkable feature of such repeat examinations has been in the reproducibility of types II and III changes in the region of the recto-sigmoid junction. Although there were variations in diameter and length of the colon, the details of the muscle bands remained constant. Type I change was also constant in its occurrence and general outline although its more complex nature did not allow such close comparison as could be effected with types II and III. Comparisons between repeat barium enema examinations on the one patient did not show the same degree of reproducibility. resection for diverticulitis two years previously showed persisting diverticula in the portion of the bowel corresponding to the descending colon. Two cases showed no evidence of muscle abnormality, while the third showed three deep type I bands immediately below the site of anastomosis (Fig. 7). None of the three patients has had any bowel symptoms after resection.

\section{Discussion}

TYPE I ABNORMALITY

The findings in this investigation (Table I) confirmed the conclusions reached in a necropsy study (Hughes, 1968) that the most characteristic muscular change in diverticular disease is the presence of bands of circular muscle, closely 
alternating from side to side, in the sigmoid colon. The incidence of type I abnormality in this series $(54 \%)$ is higher than that in the necropsy series $(31 \%)$, but this is not surprising since the necropsy series was unselected, while all patients in the present series had some bowel symptom. However, the incidence of type I muscle abnormality in those patients with diverticula $(72 \%)$ was remarkably similar to the corresponding figure in the necropsy series $(66 \%)$. This similarity of incidence in the two series suggests that the relatively early examples of muscle change detected by a functional study such as this one are accompanied by histologically recognizable structural changes.

The results in the three cases examined after sigmoid resection show that this operation as routinely practised may not entirely eliminate the type I abnormality. In this series, two patients who showed no type I remnant were treated for vesico-colic fistula and therefore may have had more extensive resection than the third patient, who had residual muscle bands after resection for acute diverticulitis. Furthermore, the distance from the anal verge to the lower limit of type I abnormality varies from approximately $22 \mathrm{~cm}$ to $35 \mathrm{~cm}$, so that a standard distal limit of resection may not encompass the muscle change in all cases. At present none of the three patients has symptoms, and it remains to be seen if symptoms appear in the future.

\section{TYPE II ABNORMALITY}

Tables I and II show a significant relationship between type II abnormality and diverticulosis, and between type II and type I abnormalities. As well as this statistical relationship, the fact that type II abnormality is frequently present in continuity with type I, the occasional occurrence of type II abnormality alone in association with diverticulosis, and the findings of transitional forms between types I and II, all suggest that type II abnormality is a part of diverticular disease. Indeed, the transitional form shown in Fig. 4 suggests a possible mechanism for the origin of type I abnormality, simple 'sigmoid' curves being converted into a precursor of type I change by longitudinal spasm. This state might possibly lead to some mechanical advantage for certain bands of fibres, leading to work hypertrophy and the typical circular muscle bands. Should this hypothesis be correct, the origin of type II abnormality itself would still require an explanation.

The question arises as to whether type II abnormality corresponds to any of the histological muscle changes in diverticular disease. If the type II functional abnormality seen here is accompanied by structural changes, it would most probably be seen histologically as a uniform thickening, and in fact uniform muscle thickening was seen in the necropsy series in about $10 \%$ of cases of diverticular muscle abnormality, the remaining $90 \%$ showing the more typical alternating muscle bands (Hughes, 1968).

\section{TYPE III ABNORMALITY}

There is no statistical evidence from this study that type III abnormality is a part of diverticular disease. However, the bands of this abnormality are striking when present, and as they are not seen anywhere in the left colon, they may have some significance. They are deeper and broader than similar changes resulting from infolding of the bowel wall at other sites of angulation of the colon, and their appearance suggests that they might have a sphincteric action.

Functionally it would seem that there must be a sphincter in the region of the recto-sigmoid, because of the way in which faeces are held in the sigmoid before defaecation. A lateral indentation in the region of the recto-sigmoid junction is often found to have a considerable obstructing effect on retrograde passage of a sigmoidoscope, and sphincter-like contractors in the uppei and middle portions of the sigmoid and at the recto-sigmoid junction have long been described by radiologists (Templeton, 1960). Attempts to demonstrate such sphincters by pressure studies have so far failed, although Shagiroff believed that muscular thickening at the recto-sigmoid junction could be detected histologically in $80 \%$ of cadavers (Sha firoff, 1956).

However, a circular sphincter would not be necessary to produce a sphincteric action in relation to solid faeces. Partial occlusion, and, in particular, a shelf-like effect produced by a laterally disposed muscle band, would impede the passage of a large bolus of formed faeces but allow the free passage of fluid of flatus. Such a mechanism would not necessarily produce any increased pressure as detected by manometric studies. Williams (1967) has shown that the passage of a mass peristaltic wave (the normal mechanism for moving faeces into the rectum and initiating defaecation) will result in the abolition of even gross type I bands, possibly by a mechanism of active relaxation. This could presumably extend to include types II and III bands lower in the colon, and if these type III bands do in fact represent a sphincter, such might be the mechanism for overcoming the 'sphincteric' action.

RELEVANCE OF MUSCULAR CHANGE TO OPERATIONS FOR DIVERTICULAR DISEASE Sigmoid resection

Patients who have undergone resection for diverticulitis are usually cured of inflammatory disease, but a considerable proportion continue to have minor symptoms of abdominal discomfort and bowel disturbance (Bolt and Hughes, 1966). Such symptoms could be due to 
failure to eliminate types I and II abnormalities in cases where these extend more distally than usual. In addition, overactivity of type III bands might cause symptoms of a functional type, and it is most unlikely that the distally situated type III bands would be included in a standard sigmoid resection. A study of the relationship between bowel symptoms and the different patterns of muscular abnormality is at present in progress in an attempt to elucidate this problem.

\section{Sigmoid myotomy}

This operation would deal with types I and II abnormality provided it was extended well down onto the rectum, but the bands of type III abnormality would escape division by an anterior myotomy because of their lateral disposition. A more complete knowledge of the anatomy and physiology of the rectum and sigmoid in health and disease is desirable before the performance of myotomy can be confidently recommended for 'functional' colonic symptoms.

I should like to thank Professor William Burnett for his interest and advice throughout this investigation. Miss Joy Cane, BSc, assisted with the statistical analyses. The Sigmoidform enemas have been supplied by Travenol Laboratories, Morton Grove, Illinois.
References

Bolt, D. E., and Hughes, L. E. (1966). Diverticulitis: a follow-up of 100 cases. Brit. med.J., 1, 1205-1209.

Cook, G. B., and Margulis, A. R. (1961). The silicone foam diagnostic enema. I. An assessment with surgical specimens of human sigmoid colon. Surgery, 50, 513-518.

Hughes, L. E. (1968). A post-mortem survey of diverticular disease of the colon. II. The muscular abnormality in the sigmoid colon. Gut, 10, 344-351.

Morson, B. C. (1963). The muscle abnormality in diverticular disease of the sigmoid co'on. Brit. J. Radiol., 36, 385-392.

Reilly, M. (1966). Sigmoid myotomy. Brit. J. Surg., 53, 859-863.

Shafiroff, B. G. P. (1956). Applied anatomy of the colon and rectum. Pediat. Clin. N. Amer., 3, 3-13.

Templeton, A. W. (1960). Colon sphincters simulating organic disease. Radiology, 75, 237-241.

Williams, I. (1967). Mass movements (mass peristalsis) and diverticular disease of the colon. Brit. J. Radiol. 40 2-14. 